\title{
Primary Cardiac Angiosarcoma. A Therapeutical Dilemma
}

\author{
José Dario Frota Filho, Fernando A. Lucchese, Paulo Leães, Luís Antônio Valente, \\ Mariana S. Vieira, Celso Blacher
}

Porto Alegre, RS - Brazil

\begin{abstract}
Cardiac angiosarcomas are malignant tumors that almost invariably have a short and fatal evolution. The therapeutic approach includes surgery, chemotherapy, and radiation therapy, alone or in combination. Heart transplantation is an attractive option in nonresectable tumors, even though the current experience is still limited. However, in most patients, the diagnosis is still established late, and survival is only slightly altered by the proposed treatments, mainly due to previously existing and undetected metastases. We report a case that illustrates the therapeutic dilemma faced with this neoplasia, and we discuss the case based on a literature review.
\end{abstract}

Approximately $25 \%$ of all primary cardiac tumors have malignant characteristics and an invasive behavior. Sarcomas account for almost all these malignant tumors, which rank second in the general incidence of cardiac tumors, right after myxomas. Sarcomas derive from the mesenchymal tissue and have a variable morphology, which makes possible their classification as angiosarcomas, rhabdomyosarcomas, fibrosarcomas, and others. From the clinical point of view, they have a rapid and fatal evolution, and the surgical results remain unsatisfactory, regardless of the techniques used. Surgery, however, allows the differential diagnosis between benign and malignant neoplasia, which is not always possible through invasive and noninvasive diagnostic examinations. Even the therapeutic combinations involving surgery and radiation therapy or chemotherapy, or both, have not proved to be effective in managing cardiac sarcomas.

\section{Case Report}

The patient was a 74-year-old female who presented to the emergency service with acute pulmonary edema. The

Hospital São Francisco - Santa Casa de Porto Alegre

Mailing address: José Dario Frota Filho - Rua Prof. Freitas Cabral, 305/602 90690-130 - Porto Alegre, RS - E-mail: dariofrota@cardiol.br

English version by Stela Maris C. e Gandour patient complained of progressive dyspnea on exertion over the past few months, prior to which she reported being healthy. The patient denied rheumatic and cardiovascular diseases. The physical examination was compatible with moderate mitral and aortic stenosis, and functional class IV. Radiography of the chest, heart, and great vessels showed an elongated aorta with parietal calcifications, in addition to small left pleural effusion. The coronary arteries were normal on coronary angiography, and atrial fibrillation was evidenced on electrocardiography.

On transthoracic and transesophageal echocardiography, a lobulated mass of large dimensions, attached to the interatrial septum was evidenced. This mass slid through the mitral valve to the left ventricular cavity also filling the left atrial auricle. Maximum and medium mitral gradients were 44 and $22 \mathrm{mmHg}$, respectively. Global systolic function was preserved with an ejection fraction above $70 \%$. The aortic valve had calcifications and a systolic flow with maximum and medium gradients of 67 and $40 \mathrm{mmHg}$, respectively. The tricuspid valve had a mild regurgitation jet and a systolic pressure in the pulmonary artery of $64 \mathrm{mmHg}$. Fibrinous pleural and small pericardial effusions were observed. These findings were suggestive of left atrial myxoma with functional mitral stenosis.

The intraoperative findings showed serosanguineous effusion in the pericardium with a large amount of fibrin (fig. 1) and massive thrombosis in the left atrium and left atrial auricle(fig. 2) with projection of the thrombi to the mitral orifice. An excessively mobile, solid, whitish, pediculate tumoral mass of hard consistency and narrow base was found attached to the mitral posterior leaflet in its basal portion (almost on top of the posterior ring) measuring approximately $4 \times 3 \mathrm{~cm}$ (fig. 3). The mitral orifice and leaflets were morphologically normal, and a functional stenosis caused by the simultaneous projection of the tumor and thrombi into the valvular orifice could be seen. Through a biatrial incision associated with an interatrial septotomy, we performed a left atrial thrombectomy and resection of the tumor attached to the mitral posterior leaflet in association with a small extension of native annulovalvar tissue. Mitral 


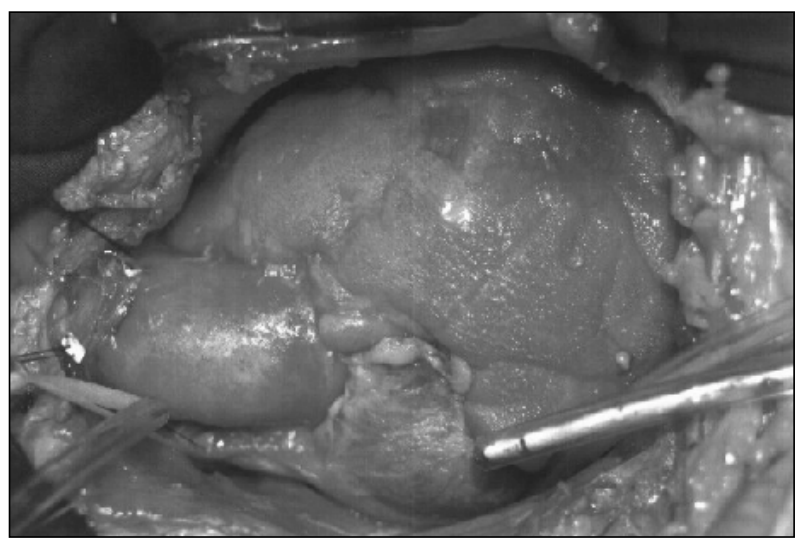

Fig. 1 - View of the pericardium recovered by a fibrin net resulting from sanguineous effusion.

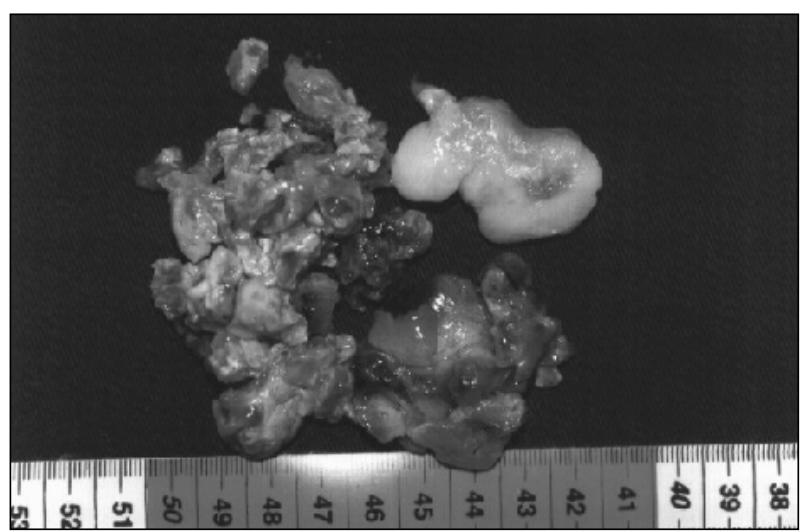

Fig. 2 - Posterior portion of the angiosarcoma attached to the mitral ring and mitral posterior leaflet, evidencing a large amount of atrial thrombi.

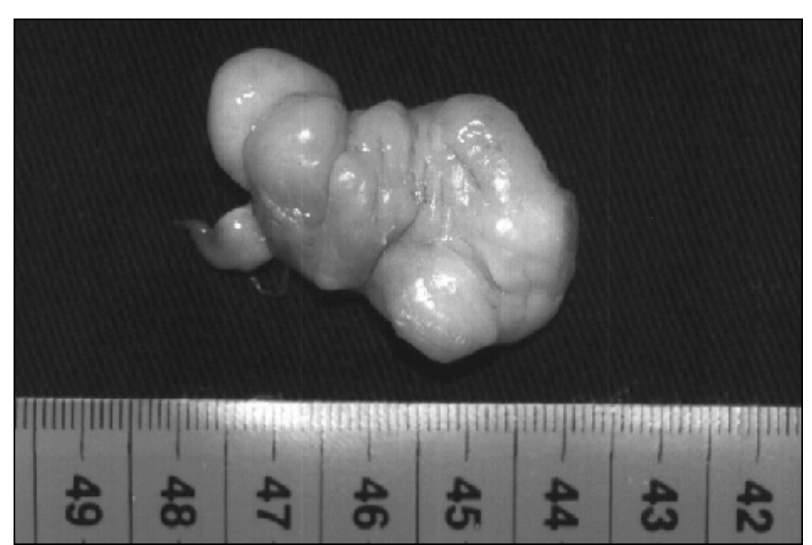

Fig. 3-Gross aspect of the anterior portion of the angiosarcoma, in which its pedicle of implantation in the mitral posterior ring can be seen.

valvoplasty (annuloplasty), aortic valvoplasty (decalcification of the 3 leaflets), and exclusion of the left atrial auricle with inner ligation were also performed.

The analysis of the pericardial effusion was negative for bacteria and tumor cells, revealing only erythrocytes $\left(90,000 / \mathrm{mm}^{3}\right)$ and othercells $\left(100 / \mathrm{mm}^{3}\right)$.

The anatomicopathological examination revealed a high-grade malignant epithelioid neoplasia with a high mitotic index and nuclear pleomorphism. The immunohistochemistry profile showed focal expression of CD31 and intense positivity of vimentin and type IV collagen in the neoplastic cells, indicating a high-grade sarcoma, angiosarcoma (fig. 4).

The postoperative transesophageal echocardiography showed the mitral valve with a normal opening, maximum and medium gradients of 10 and $3 \mathrm{mmHg}$, respectively, with no regurgitation. The aortic valve maximum and medium gradients were 20 and $9 \mathrm{mmHg}$, respectively. The left atrium was slightly enlarged $(44 \mathrm{~mm})$, but no masses were present inside. The ejection fraction was $80 \%$. No examinations to detect cerebral and pulmonary metastases were performed. The early evolution was uneventful, and the patient was discharged from the hospital in good condition and using oral anticoagulants. The patient evolved asymptomatically, and, 6 months afterwards, she died suddenly at home, probably due to ventricular arrhythmia.

\section{Discussion}

Primary cardiac tumors are not frequent. The incidence of primary cardiac neoplasias ranges from 0.001 to $0.03 \%$ in autopsy reports ${ }^{1}$. In adults, approximately $75 \%$ of the tumors are benign, the most common being myxoma, and the remaining 25\% are malignant. Angiosarcoma accounts for at least one third of the latter, is the most frequent malignant primary cardiac tumor, being reported in patients aged from 9 to 80 years, in an approximate proportion of 2 males to every female ${ }^{2,3}$. Angiosarcomas manifest mainly as a pediculate intracavitary formation in the right atrial chamber, but they may also originate in the left atrial wall ${ }^{3}$. They are characteristically lobulated masses, with necrotic foci, ranging from 2 to $30 \mathrm{~cm}$ in size, and may extend beyond the epicardium, reaching the pericardial sac. Microscopically, they comprise anastomotic vascular channels formed by malignant cells, solid areas of spindle cells, and other areas of primarily anaplastic cells ${ }^{4}$.

The natural history of cardiac angiosarcomas is characterized by a short clinical course and fatal evolution,

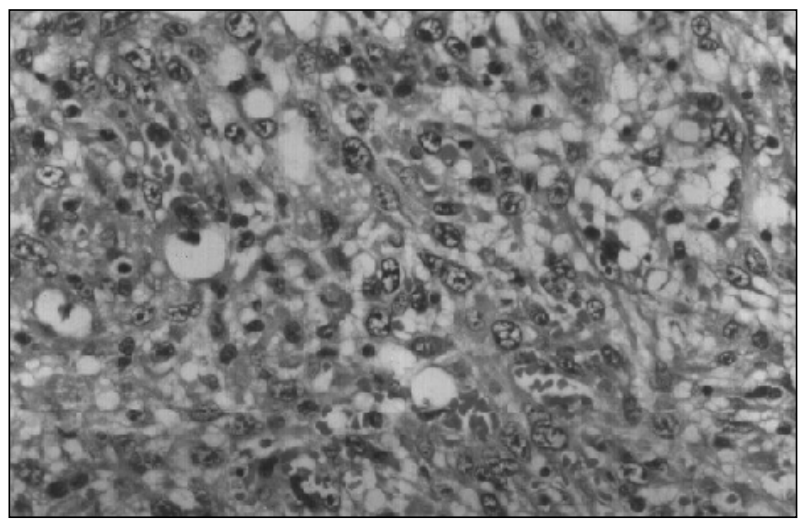

Fig. 4 - Microscopy of the sarcoma: undifferentiated epithelioid cells, mitotic figures, nuclear atypia, intracytoplasmic vacuoles, vascular formations, and polymorphonuclear leukocytes. 
even after surgery, chemotherapy, and radiation therapy ${ }^{1}$. A review of the literature shows that this is an aggressive tumor with $80 \%$ of the patients with metastases at the time of diagnosis and $90 \%$ of them surviving less than 9 months after the diagnosis (mean ranging from 6 to 11 months ${ }^{1}$ ). In the past, the diagnosis was established only on autopsy in $55 \%$ to $88 \%$ of the cases ${ }^{4}$. The clinical presentation is unspecific and relates to the location of the primary tumor, degree of local involvement, and presence or absence of metastases, which are mainly pulmonary. All these features lead to late diagnosis. Initial signs and symptoms may suggest pericarditis, with pleuritic chest pain, pericardial friction, and nonspecific electrocardiographic alterations ${ }^{4}$. Cytology of the thoracocentesis and pericardiocentesis fluids or of the biopsy may provide the diagnosis of tumor malignancy ${ }^{5}$. The signs and symptoms may include fever, dyspnea, cardiac murmurs, weight loss, orthopnea, fatigue, anorexia, vomiting, peripheral edema, and right heart failure ${ }^{3}$. Extrinsic tumoral compressions in the venae cava and obstructions of the valvular orifices lead to hepatomegaly and jugular venous distention. Hemoptysis and cardiac tamponade usually represent catastrophic events in the course of disease. Electrocardiographic alterations, such as elevation of the ST segment, inversion of the T wave, low-voltage QRS, left axial deviation, right bundle-branch block, and atrial fibrillation, are frequent findings. From the radiological point of view, we may observe cardiomegaly, mediastinal distortions secondary to invasion of the pericardial sac by tumor or hemorrhage ${ }^{4}$, and abnormalities in the pulmonary fields, a consequence of metastases. The differential diagnosis is necessary, not only between primary and metastatic, malignant and benign cardiac tumors, but also between neoplastic or nonneoplastic masses ${ }^{6}$. Imaging techniques, such as echography, tomography, magnetic resonance imaging, and angiography allow the rapid recognition of these neoplasias. The advanced stage and the dimensions of this tumor are limiting factors of successful surgical treatment. In case of tumors confined to the atrial free wall, the interatrial septum, or to a small portion of the ventricle or of a cardiac valve, complete tumor resection should be performed, in an attempt to attenuate symptoms and increase postoperative survival ${ }^{7,3}$. Often, the tumor is nonresectable due to the extension of myocardial invasion ${ }^{1}$. In these cases, adjuvant chemotherapy and radiation therapy have been frequently used. More recently, cardiac transplantation has become an alternative; most of the time, however, survival does not differ from that in which transplantation is not performed ${ }^{1}$. A hypothesis to explain the unaltered mortality after transplantation is the possibility that immunosuppressive treatment causes dissemination of preexisting micrometastases not detected at the time of diagnosis ${ }^{8}$. On the other hand, several studies emphasize cardiac transplantation as the treatment of choice for patients with nonresectable tumors, locally aggressive but with no metastases. Chemotherapy and radiation therapy neither alleviate symptoms as surgery does nor seem to prolong patients' survival ${ }^{3}$. The possibility of a treatment combining these therapeutic modalities has already been reported by Baay et al ${ }^{9}$. In that case, the patient underwent initial chemotherapy with doxorubicin, dacarbazine, ifosfamide, and mesna, which was complemented with a total dose of radiation of $2600 \mathrm{cGy}$, being then transplanted. Two months later, the patient received 2 additional courses of chemotherapy with the same drugs and was maintained on cyclosporine and prednisone. The clinical outcome was favorable, and metastases were not detected up to 33 months after surgery.

The aggressiveness of angiosarcoma continues to challenge the current methods of treatment, and the ideal therapy is yet to be discovered. In addition, postoperative survival seems not to significantly differ in patients treated with or without surgery ${ }^{1}$, independent of the extension of the surgical resection, or even of the transplant replacement of the organ.

\section{Acknowledgements}

We thank our colleagues Gilberto Friedman, Eraldo Lúcio, Marcela Sales, Wagner Pereira, Roberto Lobo, Ralf Stuermer, Nicasio Tanaka, Luis Vargas, Carlos Delmar, and Angela Bueno for their contribution during different phases of the study.

\section{References}

1. Kakizaki S, Takagi H, Hosaka Y. Cardiac angiosarcoma responding to multidisciplinary treatment. Int J Cardiol 1997; 62: 273-5.

2. Klima U, Wimmer-Greinecker G, Harringer W, Mair R, Grob CH, Brucke P. Cardiac angiosarcoma- a diagnostic dilemma. Cardiovasc Surg 1993; 1: 674-6.

3. Putnam JB, Sweeney MS, Colon R, Lanza LA, Frazier OH, Cooley DA. Primary cardiac sarcomas. Ann Thorac Surg 1991; 51:906-10.

4. Herrmann MA, Shankerman RA, Edwards WD, ShubC, SchaffHV. Primary cardiac angiosarcoma: a clinicopathologic study of six cases. J Thorac Cardiovasc Surg 1992; 103: 655-64.

5. Rudoff J, Slavin RE. Cardiac angiosarcoma arising in a coronary artery: angiographic and pathologic findings. Cathet Cardiovasc Diag 1995; 34: 215-8.
6. Basso C, Valente M, Poletti A, Casarotto D, Thiene G. Surgical pathology of primary cardiac and pericardial tumors. Eur J Cardiothorac Surg 1997; 12: 730-8.

7. McFadden PM, Ochsner JL. Atrial replacement and tricuspid valve reconstruction after angiosarcoma resection. Ann Thorac Surg 1997; 64: 1164-6.

8. Crespo MG, Pulpón LA, Pradas G, et al. Heart transplantation for cardiac angiosarcoma: should its indication be questioned? J Heart Lung Transplant 1993; 12 : 527-30.

9. Baay P, Karwande SV, Kushner JP, Olsen S, Renlund DG. Successful treatment of a cardiac angiosarcoma with combined modality therapy. J Heart Lung Transplant 1994; 13: 923-5. 